\title{
Ghrelin Gene Polymorphisms in Irritable Bowel Syndrome
}

\author{
Theodora Kalli ${ }^{a}$ Tilemachos Koutouratsas ${ }^{b}$ George Karamanolis ${ }^{c}$ \\ Maria Gazoulib

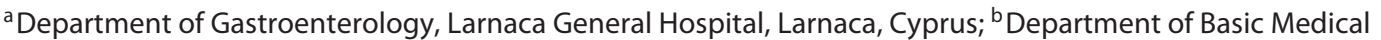 \\ Science, Laboratory of Biology, School of Medicine, National and Kapodistrian University of Athens, Athens, Greece; \\ '2nd Department of Surgery, Gastroenterology Unit, "Aretaieio" University Hospital, School of Medicine, National \\ and Kapodistrian University of Athens, Athens, Greece
}

\section{Keywords}

Irritable bowel syndrome · Ghrelin · Functional

gastrointestinal disorders $\cdot$ Diarrhea $\cdot$ Constipation

\begin{abstract}
Introduction/Objective: Irritable bowel syndrome (IBS) is a bowel disorder characterized by pain accompanying defecation or altered bowel habits, divided into diarrhea-predominant, constipation-predominant, and alternating subtypes, whose pathogenesis is considered to include disordered bowel motility. The hormone ghrelin is a growth hormone secretagogue which furthermore affects gastrointestinal motility. We study the association between its genetic polymorphisms and the risk for IBS. Methods: IBS patients meeting the Rome III criteria and controls similar in age and gender were recruited. Whole blood samples were used for genotyping via polymerase chain reaction and restriction fragment length polymorphism for the polymorphisms rs34911341, rs696217, and rs2075356. Results: Participants included 142 patients and 209 controls. The rs696217 GG genotype frequency was higher in patients $(78.87 \%)$ compared to controls (55.5\%). The rs696217 GT genotype was significantly less frequent among patients than in controls (OR $0.31,95 \% \mathrm{Cl} 0.19-0.52$ ), as was the T allele (OR 0.43 , 95\%
\end{abstract}

$\mathrm{Cl}$ 0.28-0.66). No significant differences in genotype distribution were found for the rs34911341 and rs2075356 polymorphisms between patients and controls. The genotype frequencies did not significantly differ between IBS subtype groups for any of the polymorphisms studied. Conclusions: The GG and GT genotypes of the rs696217 polymorphism, as well as the $\mathrm{G}$-allele, demonstrate significant association with IBS susceptibility, while the T allele appears to bear a protective effect. Ghrelin's polymorphisms are plausibly involved in IBS pathogenesis, but do not correlate with any distinct IBS subtype.

c) 2020 S. Karger AG, Basel

\section{Introduction}

Irritable bowel syndrome (IBS) is chronic or remittent bowel disorder characterized by abdominal pain which accompanies defecation or a change in bowel habits, in the absence of evident anatomic or physiologic abnormalities [1]. It is a symptom-defined disorder, which can be further divided into diarrhea-pre-

T. Kalli and T. Koutouratsas contributed equally to this manuscript.

Maria Gazouli, $\mathrm{PhD}$

Department of Basic Medical Sciences, Laboratory of Biology School of Medicine, National and Kapodistrian University of Athens Michalakopoulou 176, GR-11527 Athens (Greece)

E-Mail mgazouli@med.uoa.gr 
dominant IBS (IBS-D), constipation-predominant IBS (IBS-C), and alternating-pattern IBS subtypes [2]. More specifically, according to Rome III criteria, IBS is diagnosed when abdominal pain or discomfort is present for at least 3 days per month for the past 3 months, with 2 of the following characteristics: (1) relief by defecation, (2) onset associated with a change in stool frequency, and (3) onset associated with a change in stool form [3].

Many mechanisms are thought to be involved in the pathophysiology of IBS, including altered gastrointestinal motility, abnormal gas handling, altered gastrointestinal secretions, visceral hypersensitivity at the mucosal, spinal, subcortical and cortical levels, mucosal inflammation, postinfectious sequelae, alterations of enteroendocrine tissue [4], and dysregulation of the brain-gut axis [5-7]. Its etiology is most likely multifactorial, including environmental factors, psychosocial stress, and alterations in normal gut flora [7]. Genetic factors could plausibly contribute in certain cases, as a study including 11,986 twin pairs reported a higher concordance in monozygotic than in dizygotic twins [8].

Ghrelin is a peptide hormone comprising 28 amino acids which is secreted by neuroendocrine cells called $\mathrm{P} /$ D1 cells, mainly in the fundus of the stomach [9]. It exists in 2 forms in tissues and plasma: n-octanoyl-ghrelin, which is octanoylated at the Ser3 residue, and des-acyl ghrelin, which has not undergone this modification [10]. Its gene, GHRL, is located on chromosome 3p25-26 and it comprises 5 exons, including 2 different sites for initiation of transcription, at -80 and at -555 relative to the start codon [11]. Opposite to GHRL is a gene called GHRL opposite strand, whose RNA products are alternatively spliced and are thought to participate in the regulation of the ghrelin axis as noncoding RNAs [12]. At least 300 single nucleotide polymorphisms (SNPs) have been discovered in GHRL's coding and noncoding regions, 4 of which bear pathogenic associations [13].

Ghrelin's expression is mainly driven by fasting, although it is moreover negatively regulated by somatostatin, parasympathetic activation, and a diet high in fat [9]. Ghrelin is considered a primary element of the brain-gut axis [14]. Its physiologic actions include an increase in growth hormone levels, appetite stimulation, increased gastric acid secretion and motility, mucosal protection, induction of migrating motor complexes, and a decrease in blood pressure $[9,10,14]$. Liver- and small intestinederived liver-enriched antimicrobial peptide- 2 is a recently characterized component of ghrelin's physiology, acting as an endogenous antagonist of ghrelin's receptor [15].

Ghrelin's serum levels, as well as certain polymorphisms, have been associated with several phenotypes and disorders. Bodyweight and fat mass have been negatively correlated with serum ghrelin concentration in mice [16], while in humans, patients with binge eating disorder have been found to have lower fasting ghrelin levels [17], suggesting a plausible role for ghrelin in the pathophysiology of obesity. In a population of Japanese women, the T C allele of the $3056 \mathrm{SNP}$ was positively correlated with higher fasting acyl-ghrelin levels and anthropometric obesity measures [18]. Regarding gastrointestinal pathology, higher ghrelin serum levels are observed in patients with celiac disease, and in adults, its levels normalize after following a gluten-free diet [19, 20]. Higher serum ghrelin levels also correlate with disease activity in ulcerative colitis and Crohn's disease [19]. Ghrelin serum levels have been positively correlated with functional dyspepsia; however, no certain polymorphisms in the GHRL gene have exhibited a significant association with disease state [21]. Ghrelin's serum levels also appear to positively correlate with cyclic vomiting syndrome and infant colic [21].

Recently, Russo et al. [22] suggested that the GT genotype and the T allele of the GHRL rs696217 polymorphism were reduced in IBS-D patients compared to healthy individuals (HC), but only a small number of IBS-D only patients and controls were tested in this study. Additionally, Dats-Opoka et al. [23] supported that the rs696217 GG genotype is associated with a threefold increase in risk for GERD development in children. Therefore, since the relative information is currently limited, in the present study, we examined the association between the risk of IBS and certain GHRL gene polymorphisms in a well-characterized cohort of Greek IBS patients.

\section{Materials and Methods}

Subjects

One hundred forty-two Greek IBS patients fulfilling the Rome III diagnostic criteria for IBS [24] and 209 HC similar in age and gender to the patient population used as controls. All the participants enrolled were attending the Outpatient Clinic of the University Hospital. Patients and controls gave written informed consent. The study was approved by the Ethics Committee of the participating hospitals. Specifically, this study enrolled 62 patients with IBS-D, 55 patients with IBS-C, and 25 patients with mixed IBS [24]. The health status of the controls was assessed by detailed medical history, thorough physical examination, includ- 
Table 1. Characteristics of IBS patients and healthy controls

\begin{tabular}{|c|c|c|c|c|}
\hline & $\begin{array}{l}\text { IBS-C } \\
(n=55)\end{array}$ & $\begin{array}{l}\text { IBS-D } \\
(n=62)\end{array}$ & $\begin{array}{l}\text { IBS-M } \\
(n=25)\end{array}$ & $\begin{array}{l}\text { Healthy controls } \\
(n=209)\end{array}$ \\
\hline Age, years, mean $\pm S D$ & $57.9 \pm 16.2$ & $60.9 \pm 14.4$ & $58.3 \pm 12.8$ & $62.3 \pm 15.8$ \\
\hline \multicolumn{5}{|l|}{ Gender, $n(\%)$} \\
\hline Male & $23(41.8)$ & $35(56.5)$ & $13(52)$ & $106(50.7)$ \\
\hline Female & $32(58.2)$ & $27(43.5)$ & $12(48)$ & $103(49.3)$ \\
\hline \multicolumn{5}{|l|}{ Smoking, $n(\%)$} \\
\hline Yes & $12(21.8)$ & $25(40.3)$ & $10(40)$ & $79(37.8)$ \\
\hline No & $43(78.2)$ & $37(59.7)$ & $15(60)$ & $130(62.2)$ \\
\hline \multicolumn{5}{|c|}{ Family history of IBS, $n(\%)$} \\
\hline Yes & $8(14.5)$ & $5(8.1)$ & $0(0)$ & $0(0)$ \\
\hline No & $47(85.5)$ & $57(91.9)$ & $25(100)$ & $209(100)$ \\
\hline
\end{tabular}

IBS, irritable bowel syndrome; IBS-C, constipation-predominant IBS; IBS-D, diarrhea-predominant IBS; IBS-M, mixed IBS.

Table 2. PCR primers and restriction enzymes

\begin{tabular}{|c|c|c|c|}
\hline SNP & Primers $\left(5^{\prime}-3^{\prime}\right)$ & Reaction conditions & $\begin{array}{l}\text { Restriction enzyme } \\
\text { and products, bp }\end{array}$ \\
\hline rs2075356 & $\begin{array}{l}\text { F: AAGGGGGCGTTGAACTAGG } \\
\text { R: CTGAGAATGCTGGGCAGACC }\end{array}$ & $\begin{array}{l}95^{\circ} \mathrm{C} \text { for } 1 \mathrm{~min} \\
95^{\circ} \mathrm{C} \text { for } 50 \mathrm{~s} \\
60^{\circ} \mathrm{C} \text { for } 50 \mathrm{~s} \\
72^{\circ} \mathrm{C} \text { for } 50 \mathrm{~s} \\
\text { Repeat steps } 2-4 \times 40 \\
72^{\circ} \mathrm{C} \text { for } 10 \mathrm{~min}\end{array}$ & $\begin{array}{l}\text { Eco57I (acuI) } \\
\text { C: } 312,87 \\
\text { T: } 262,87,50\end{array}$ \\
\hline rs696217 & $\begin{array}{l}\text { F: GCTGGGCTCCTACCTGAGC } \\
\text { R: GGACCCTGTTCACTGCCAC }\end{array}$ & $\begin{array}{l}94^{\circ} \mathrm{C} \text { for } 5 \mathrm{~min} \\
94^{\circ} \mathrm{C} \text { for } 30 \mathrm{~s} \\
65^{\circ} \mathrm{C} \text { for } 30 \mathrm{~s} \\
72^{\circ} \mathrm{C} \text { for } 30 \mathrm{~s} \\
\text { Repeat steps } 2-4 \times 36 \\
72^{\circ} \mathrm{C} \text { for } 10 \mathrm{~min}\end{array}$ & $\begin{array}{l}\text { BsrI } \\
\text { T: } 618 \\
\text { G: } 517,101\end{array}$ \\
\hline rs34911341 & $\begin{array}{l}\text { F: GGACCCTGTTCACTGCCAC } \\
\text { R: GGACATGGGGGCTTAGAGT }\end{array}$ & $\begin{array}{l}94^{\circ} \mathrm{C} \text { for } 1 \mathrm{~min} \\
95^{\circ} \mathrm{C} \text { for } 40 \mathrm{~s} \\
59^{\circ} \mathrm{C} \text { for } 40 \mathrm{~s} \\
72^{\circ} \mathrm{C} \text { for } 40 \mathrm{~s} \\
\text { Repeat steps } 2-4 \times 40 \\
72^{\circ} \mathrm{C} \text { for } 5 \mathrm{~min}\end{array}$ & $\begin{array}{l}\text { SacI } \\
\text { T: } 258 \\
\text { C: } 163,95\end{array}$ \\
\hline
\end{tabular}

PCR, polymerase chain reaction.

ing regular laboratory tests prior to study enrollment, which confirmed the absence of any chronic gastrointestinal or psychological disease. Patients' clinical characteristics are illustrated in Table 1 .

\section{DNA Isolation and Genotyping}

DNA was extracted from whole blood samples of recruited IBS patients and healthy controls using the Nucleospin Blood QuickPure kit (Macherey-Nagel GmbH and Co. KG, Düren, Germany).
The DNA samples were then separately amplified for each SNP using polymerase chain reaction (PCR). The primers and PCR conditions for each polymorphism studied are presented in Table 2. The primers for polymorphisms rs34911341 and rs2075356 were designed using the Gene Runner version 6 molecular analysis software and primerBLAST for confirmation of specificity, whereas the primers for the rs696217 SNP were those published by Yang et al. [25]. The reaction products were then digested using restriction enzymes (Table 2 ) and analyzed on $2 \%$ agarose gel 
DNA electrophoresis in the presence of Nippon Genetics FastGene 100bp DNA ladder, using ethidium bromide as stain. The allele discrimination results were verified through sequencing of a subgroup of representative PCR products.

\section{Statistical Analysis}

Genotype frequencies were analyzed with the $\chi^{2}$ test with Yate's correction using S-Plus (version 6.2 Insightful, Seattle, WA, USA) software. ORs and 95\% CIs were calculated with GraphPad (version 300, GraphPad Software, San Diego, CA, USA). All $p$ values are two-sided. $p$ values of $<0.05$ were considered significant.

\section{Results}

Genotype and allele frequencies of the GHRL polymorphisms tested in IBS patients and $\mathrm{HC}$ are presented in Table 3. The genotypic distributions of all 3 polymorphisms in patient group and HC followed the HardyWeinberg equilibrium $(p>0.05)$. No statistically significant differences were found in genotype and allele frequency distributions regarding the rs34911341 and rs2075356 polymorphisms (Table 3 ). However, as indicated in Table $3,78.87 \%$ of patients with IBS had the rs696217 GG genotype, compared to $55.50 \%$ among individuals of the control group. Rs696217 GT genotype was registered approximately 2 times more seldom in IBS patients of the studied group comparing with the individuals from the HC group: 18.30 and $41.15 \%$, respectively $(p<0.0001)$.

Table 4 presents the distribution of rs34911341, rs2075356, and rs696217 genotypes depending on IBS phenotype (IBS-D, IBS-C, and mixed IBS). For all studied polymorphisms, no statistically significant differences in genotype frequencies between the patient groups were revealed, when distributed according to IBS subtype.

\section{Discussion}

In the present work, we present the distribution of alleles and genotypes for the rs34911341, rs2075356, and rs696217 polymorphisms of the GHRL gene in a group of patients with IBS, in comparison to their distribution among a control group of HC. As indicated by the results, the rs696217 GG and GT genotypes as well as the G-allele were significantly related to IBS susceptibility. The presence of the rs696217 T allele demonstrated a protective effect against IBS development. Our results are partly in
Table 3. Genotype and allele distributions

\begin{tabular}{cccl}
\hline & IBS, $n(\%)$ & HC, $n(\%)$ & $p$ value; OR $(95 \%$ CI $)$ \\
\hline rs2075356 & & & \\
TT & $62(43.7)$ & $77(36.9)$ & 1.00 (ref.) \\
TC & $66(46.5)$ & $105(50.2)$ & $0.29 ; 0.78(0.49-1.23)$ \\
CC & $14(9.8)$ & $27(12.9)$ & $0.28 ; 0.64(0.31-1.33)$ \\
T-allele & $190(66.9)$ & $259(62)$ & 1.00 (ref.) \\
C-allele & $94(33.1)$ & $159(38)$ & $0.20 ; 0.81(0.59-1.11)$ \\
rs696217 & & & \\
GG & $112(78.9)$ & $116(55.5)$ & $1.00($ ref.) \\
GT & $26(18.3)$ & $86(41.1)$ & $<0.0001 ; 0.31(0.19-0.52)$ \\
TT & $4(2.8)$ & $7(3.4)$ & $0.54 ; 0.59(0.17-2.08)$ \\
G-allele & $250(88)$ & $318(76.1)$ & 1.00 (ref.) \\
T-allele & $34(12)$ & $100(23.9)$ & $<0.0001 ; 0.43(0.28-0.66)$ \\
rs34911341 & & & \\
CC & $91(64.1)$ & $148(70.8)$ & 1.00 (ref.) \\
CT & $42(29.6)$ & $56(26.8)$ & $0.46 ; 1.22(0.76-1.97)$ \\
TT & $9(6.3)$ & $5(2.4)$ & $0.09 ; 2.93(0.95-9.01)$ \\
C-allele & $224(78.9)$ & $352(84.2)$ & 1.00 (ref.) \\
T-allele & $60(21.1)$ & $66(15.8)$ & $0.07 ; 1.43(0.97-2.10)$ \\
\hline
\end{tabular}

IBS, irritable bowel syndrome; HC, healthy individuals.

Table 4. Distribution of GHRL rs2075356, rs696217, and rs34911341 genotypes in IBS-D, IBS-C, and IBS-M cases

\begin{tabular}{lcccc}
\hline & $\begin{array}{c}\text { IBS-D } \\
(n=62)\end{array}$ & $\begin{array}{c}\text { IBS-C } \\
(n=55)\end{array}$ & $\begin{array}{c}\text { IBS-M } \\
(n=25)\end{array}$ & $p$ value \\
\hline rs2075356, $n(\%)$ & & & & \\
TT & $25(40.3)$ & $21(38.2)$ & $16(64)$ & \\
TC & $28(45.2)$ & $31(56.4)$ & $7(28)$ & 0.08 \\
CC & $9(14.5)$ & $3(5.4)$ & $2(8)$ & \\
rs696217, $n(\%)$ & & & & \\
GG & $48(77.4)$ & $46(83.6)$ & $18(72)$ & \\
GT & $12(19.4)$ & $7(12.7)$ & $7(28)$ & 0.48 \\
TT & $2(3.2)$ & $2(3.7)$ & 0 & \\
rs34911341, $n(\%)$ & & & & \\
CC & $38(61.3)$ & $33(60.0)$ & $20(80)$ & \\
CT & $19(30.6)$ & $19(34.5)$ & $4(16)$ & 0.43 \\
TT & $5(8.1)$ & $3(5.5)$ & $1(4)$ & \\
\hline
\end{tabular}

IBS-C, constipation-predominant IBS; IBS-D, diarrhea-predominant IBS; IBS-M, mixed IBS.

agreement with a previous study by Russo et al. [22] that reported a reduction of both the rs696217 GT genotype and the $\mathrm{T}$ allele in IBS-D patients, given that when we classified our patients according to IBS subtype, no statistically significant differences were observed for any of the polymorphisms tested. Our results regarding rs696217 are in agreement with the previous study on GERD by Dats-Opoka et al. [23]. 
Regarding the rs34911341 and rs2075356 polymorphisms, no statistically significant association with IBS was observed in this present study. Our results are in agreement with those reported by Russo et al. [22]; additionally, studies on GHRL rs34911341 and rs2075356 gene polymorphisms and their relationship to components of the metabolic syndrome or diabetes mellitus type 2 did not reveal any significant associations [26, 27].

It must be noted that no study of the GHRL gene among Greek patients with IBS had been previously carried out; we therefore first report allele frequencies for the rs34911341, rs696217, and rs2075356 loci of the GHRL gene in this population. Other studies studying allele frequencies for the rs696217 polymorphism in the European region have reported a lower population frequency for the T allele (6.5-8.8\% compared to $23.9 \%$ reported in the present study). The frequency for the $\mathrm{T}$ allele found in this study is closer to the one (19.1\%) reported in East Asian populations [28]. One plausible explanation for the discrepancy between these results is that these studies have focused on European populations, but not on the Balkans [26, 28-30]; the present study is the first to report rs696217 allele frequencies among Greek healthy controls.

As previously noted, ghrelin is known to increase motility in the stomach and small intestine, without a similar effect on the colon, whereas ghrelin agonists which additionally enter the CNS can increase colonic contractility and induce defecation $[9,11,14,31,32]$. Ghrelin could plausibly play a role in the pathophysiological events leading to IBS, as a component of the model describing disturbances in gastrointestinal motility [5-7].

Researchers have previously observed significant correlations between IBS and plasma ghrelin levels or ghrelin-producing cell density in stomach tissue samples. ElSalhy et al. [33] have reported ghrelin-producing cell density to be significantly higher in IBS-D and significantly lower in IBS-C patients compared to controls, as observed in stomach biopsy samples, without similar significant findings for ghrelin plasma levels. ŞahinEryılmaz et al. [34] found higher plasma ghrelin levels in patients with IBS-D and higher ghrelin staining in tissue samples from patients with IBS-C, in comparison to controls.

Consequently, one mechanism explaining the putative role for the examined intronic [27] SNPs in IBS pathogenesis is through alteration of ghrelin's expression and total circulating levels. However, the rs2075356 and rs696217 polymorphisms have not been shown to significantly affect plasma ghrelin levels [27]; therefore, their role in the emergence of IBS, if any, would likely involve different molecular events. No similar data is currently available for the rs34911341 polymorphism.

\section{Conclusions}

Our results support that the rs696217 polymorphism is associated with IBS susceptibility. However, further research, involving larger sample sizes, examining IBS subtypes, employing qPCR for calculation of GHRL mRNA and separate measurements of its acylated and desacylated forms, and including other GHRL polymorphisms, is certainly required to elucidate its contribution to the disease's pathogenesis. A distinct focus on the newly characterized liver-enriched antimicrobial peptide-2 [15] peptide's interaction with the ghrelin axis in the setting of disease could also clarify aspects of ghrelin's pathophysiology not previously explored.

\section{Acknowledgments}

None.

\section{Statement of Ethics}

The study is in accordance with the World Medical Association Declaration of Helsinki. All the participants have given their written informed consent, and the study protocol was approved by our institute committee on human research.

\section{Disclosure Statement}

The authors have no conflicts of interest to disclose.

\section{Author Contributions}

We certify that all authors have contributed to and agreed on the content of the manuscript. M.G. and G.K. conceived of the presented idea; M.G. designed the study; T. Kalli and T. Koutouratsas performed the experiments and wrote the article with support from M.G. and G.K.; T. Kalli and G.K. contributed to data collection; T. Koutouratsas and M.G. contributed to the interpretation of the results; and M.G. and G.K. made the critical revision of the article and the final approval of the version to be published. 


\section{References}

1 Mearin F, Lacy BE, Chang L, Chey WD, Lembo AJ, Simren M, et al. Bowel Disorders. Gastroenterology. 2016 Feb;pii:S0016-5085(16)00222-5.

2 Chey WD, Kurlander J, Eswaran S. Irritable bowel syndrome: a clinical review. JAMA. 2015 Mar;313(9):949-58.

3 Clarke G, Quigley EM, Cryan JF, Dinan TG. Irritable bowel syndrome: towards biomarker identification. Trends Mol Med. 2009 Oct; 15(10):478-89.

4 El-Salhy M, Hatlebakk JG, Hausken T. Possible role of peptide YY (PYY) in the pathophysiology of irritable bowel syndrome (IBS). Neuropeptides. 2020 Feb;79:101973.

5 Posserud I, Ersryd A, Simrén M. Functional findings in irritable bowel syndrome. World J Gastroenterol. 2006 May;12(18):2830-8.

6 Clark C, DeLegge M. Irritable bowel syndrome: a practical approach. Nutr Clin Pract. 2008 Jun-Jul;23(3):263-7.

7 Karantanos T, Markoutsaki T, Gazouli M, Anagnou NP, Karamanolis DG. Current insights in to the pathophysiology of Irritable Bowel Syndrome. Gut Pathog. 2010 May;2(1):

8 Levy RL, Jones KR, Whitehead WE, Feld SI, Talley NJ, Corey LA. Irritable bowel syndrome in twins: heredity and social learning both contribute to etiology. Gastroenterology. 2001 Oct;121(4):799-804.

9 Inui A, Asakawa A, Bowers CY, Mantovani G, Laviano A, Meguid MM, et al. Ghrelin, appetite, and gastric motility: the emerging role of the stomach as an endocrine organ. FASEB J. 2004 Mar;18(3):439-56.

10 Kojima M, Kangawa K. Ghrelin: structure and function. Physiol Rev. 2005 Apr;85(2) 495-522.

11 Sato T, Nakamura Y, Shiimura Y, Ohgusu H, Kangawa K, Kojima M. Structure, regulation and function of ghrelin. J Biochem. $2012 \mathrm{Feb}$ 151(2):119-28

12 Seim I, Carter SL, Herington AC, Chopin LK Complex organisation and structure of the ghrelin antisense strand gene GHRLOS, a candidate non-coding RNA gene. BMC Mol Biol. 2008 Oct;9(1):95.

13 Ghrl - SNP - NCBI
14 Peeters TL. Ghrelin: a new player in the control of gastrointestinal functions. Gut. 2005 Nov;54(11):1638-49.

15 Mani BK, Puzziferri N, He Z, Rodriguez JA, Osborne-Lawrence S, Metzger NP, et al. LEAP2 changes with body mass and food intake in humans and mice. J Clin Invest. 2019 Sep;129(9):3909-23.

16 Korbut AI, Klimontov VV, Orlov NB Khotskina AS, Zav'yalov EL. Relationships between Body Composition and Plasma Levels of Pancreatic, Gut, and Adipose Tissue Hormones in $\mathrm{db} / \mathrm{db}$ Mice, a Model of Type 2 Diabetes Mellitus. Bull Exp Biol Med. 2019 Jul;167(3):325-8

17 Geliebter A, Gluck ME, Hashim SA. Plasma ghrelin concentrations are lower in binge-eating disorder. J Nutr. 2005 May;135(5):132630 .

18 Ando T, Ichimaru Y, Konjiki F, Shoji M, Komaki G. Variations in the preproghrelin gene correlate with higher body mass index, fat mass, and body dissatisfaction in young Japanese women. Am J Clin Nutr. 2007 Jul;86(1): 25-32.

19 El-Salhy M. Ghrelin in gastrointestinal diseases and disorders: a possible role in the pathophysiology and clinical implications (review). Int J Mol Med. 2009 Dec;24(6):727-32.

20 Cheung CK, Wu JC. Role of ghrelin in the pathophysiology of gastrointestinal disease. Gut Liver. 2013 Sep;7(5):505-12.

21 Koutouratsas T, Kalli T, Karamanolis G, Gazouli M. Contribution of ghrelin to functional gastrointestinal disorders' pathogenesis. World J Gastroenterol. 2019 Feb;25(5): 539-51.

22 Russo F, Chimienti G, Linsalata M, Clemente C, Orlando A, Riezzo G. The obestatin/ghrelin ratio and ghrelin genetics in adult celiac patients before and after a gluten-free diet, in irritable bowel syndrome patients and healthy individuals. Eur J Gastroenterol Hepatol. 2017 Feb;29(2):160-8.

23 Dats-Opoka M, Makukh H, Hnateyko OP. Leu72met Mutation of Ghrelin Ghrl Gene in Children with Gastroesophageal Reflux Disease. EUREKA: Health Sciences. 2017;3:10-7.
24 Drossman DA. The functional gastrointestinal disorders and the Rome III process. Gastroenterology. 2006 Apr;130(5):1377-90.

25 Yang Y, Li W, Zhao J, Zhang H, Song X, Xiao $\mathrm{B}$, et al. Association between ghrelin gene (GHRL) polymorphisms and clinical response to atypical antipsychotic drugs in Han Chinese schizophrenia patients. Behav Brain Funct. 2012 Feb;8(1):11.

26 Mora M, Adam V, Palomera E, Blesa S, Díaz G, Buquet X, et al.; Mataró Aging Study Group. Ghrelin Gene Variants Influence on Metabolic Syndrome Components in Aged Spanish Population. PLoS One. 2015 Sep 10(9):e0136931.

27 Joatar FE, Al Qarni AA, Ali ME, Al Masaud A, Shire AM, Das N, et al. Leu72Met and Other Intronic Polymorphisms in the GHRL and GHSR Genes Are Not Associated with Type 2 Diabetes Mellitus, Insulin Resistance, or Serum Ghrelin Levels in a Saudi Population. Endocrinol Metab (Seoul). 2017 Sep;32(3):360-

28 Genomes Project 1000 Genomes Project phase 3 release $\mathrm{V} 3+$. EBI2014.

29 Genetic variation in the Estonian population. 2018. Available from: https://www.ncbi.nlm. nih.gov/bioproject/PRJNA489787.

30 Huang R, Tian S, Cai R, Sun J, Shen Y, Wang S. Ethnicity-Specific Association Between Ghrelin Leu72Met Polymorphism and Type 2 Diabetes Mellitus Susceptibility: An Updated Meta-Analysis. Front Genet. 2018 Nov;9:541.

31 Tack J, Depoortere I, Bisschops R, Delporte C, Coulie B, Meulemans A, et al. Influence of ghrelin on interdigestive gastrointestinal motility in humans. Gut. 2006 Mar;55(3):327-33.

32 Veldhuis JD, Bowers CY. Integrating GHS into the Ghrelin System. Int J Pept. 2010;2010 pii:879503.

33 El-Salhy M, Lillebø E, Reinemo A, Salmelid L. Ghrelin in patients with irritable bowel syndrome. Int J Mol Med. 2009 Jun;23(6):703-7.

34 Sahin-Eryilmaz G, Basak K, Cakir-Madenci O, Koc H, Tuzun S, Dolapcioglu C, et al. Relationship between irritable bowel syndrome and plasma and tissue ghrelin levels. Turk J Gastroenterol. 2018 Nov;29(6):631-5. 